\title{
Gastrointestinal bleeding due to pancreatic metastasis of non-small cell lung cancer: A report of two cases and a literature review
}

\author{
YULONG ZHENG ${ }^{1}$, QIQI GAO ${ }^{2}$, WEIJIA FANG ${ }^{1}$, NONG XU $^{1}$ and JIANYING ZHOU ${ }^{3}$ \\ Departments of ${ }^{1}$ Medical Oncology, ${ }^{2}$ Pathology and ${ }^{3}$ Respiratory Medicine, \\ The First Affiliated Hospital of Zhejiang University, Hangzhou, Zhejiang 310003, P.R. China
}

Received February 22, 2014; Accepted November 13, 2014

DOI: $10.3892 / \mathrm{ol} .2015 .3035$

\begin{abstract}
The current study presents two cases of gastrointestinal (GI) bleeding due to pancreatic metastasis of non-small cell lung cancer (NSCLC). The two patients had a history of lung adenocarcinoma prior to pancreatic metastasis. The first patient presented with melena, epigastric pain and weight loss, and the second patient presented with melena and weight loss. The diagnoses were confirmed by biopsy of the metastatic lesions. Immunohistochemistry is useful for managing the differential diagnosis between lung cancer and pancreatic carcinoma in patients with a history of lung cancer. Chemotherapy prolonged the survival of the second patient. However, the first patient received no chemotherapy and succumbed to NSCLC six weeks after the diagnosis of metastatic pancreatic cancer. GI bleeding as a result of pancreatic metastasis of NSCLC is extremely rare; including the present two cases, only three cases have been reported in the English literature. The current study is presented in order to increase the awareness of GI hemorrhage as a manifestation of this relatively rare condition.
\end{abstract}

\section{Introduction}

Non-small cell lung cancer (NSCLC) accounts for $~ 85 \%$ of all lung cancers, worldwide (1). In total, $40 \%$ of newly diagnosed NSCLC cases are classified as stage-IV disease, with distant metastasis already present. However, even in patients with early-stage disease, the recurrence rate is high. Metastasis to any organ in the body may occur, but the adrenal glands, liver, brain and bones are the most common sites (2). Pancreatic involvement secondary to lung cancer is not uncommon (3-5),

Correspondence to: Professor Jianying Zhou, Department of Respiratory Medicine, The First Affiliated Hospital of Zhejiang University, 79 Qingchun Road, Hangzhou, Zhejiang 310003, P.R. China

E-mail: zjyhz@zju.edu.cn

Key words: non-small cell lung cancer, gastrointestinal bleeding, pancreas, metastasis however, metastasis of tumors to the pancreas rarely becomes clinically evident, and is usually incidentally identified during abdominal imaging in the follow-up period. Gastrointestinal (GI) bleeding as a clinical presentation of the pancreatic metastasis of lung cancer has rarely been reported (6).

In the present study, two cases of lung cancer metastasis to the pancreas that presented with upper GI bleeding are reported. To the best of our knowledge, this is the second study concerning GI bleeding caused by the pancreatic metastasis of lung cancer. The present study may increase the awareness of GI hemorrhage as a manifestation of this relatively rare condition. This study was approved by the Ethics Committee of The First Affiliated Hospital of Zhejiang University. Written informed consent was obtained from the patients' family for both patients.

\section{Case report}

Case one. In June 2013, a 56-year-old male was diagnosed with right upper lung adenocarcinoma [pT2aN0M0; stage IB, according to the American Joint Committee on Cancer (AJCC) TNM staging system (7)] and underwent thoracoscopic lobectomy at The First Affiliated Hospital of Zhejiang University (Hangzhou, China). Pathological examination revealed high-grade adenocarcinoma (Fig. 1A), with clear surgical margins and negative lymph nodes. Immunostaining revealed positivity for thyroid transcription factor-1 (TTF-1) (Fig. 1B) and cytokeratin (CK)7, and negativity for p63 and CK5/6. No evidence of distant metastasis was detected. The patient was treated with adjuvant chemotherapy using a platinum-based combined regimen, which was administered at another hospital.

The patient presented again to The First Affiliated Hospital of Zhejiang University four months later (October, 2013) with melena, epigastric pain and weight loss of $5 \mathrm{~kg}$. The patient had no history of pulmonary distress or dizziness, and the medical history was unremarkable, with no additional smoking history. The patient's performance status was 1, according to The Eastern Cooperative Oncology Group (8). A physical examination revealed anemia and upper abdominal tenderness. The patient's blood pressure was $137 / 83 \mathrm{mmHg}$ and the pulse rate was 76 beats per minute 

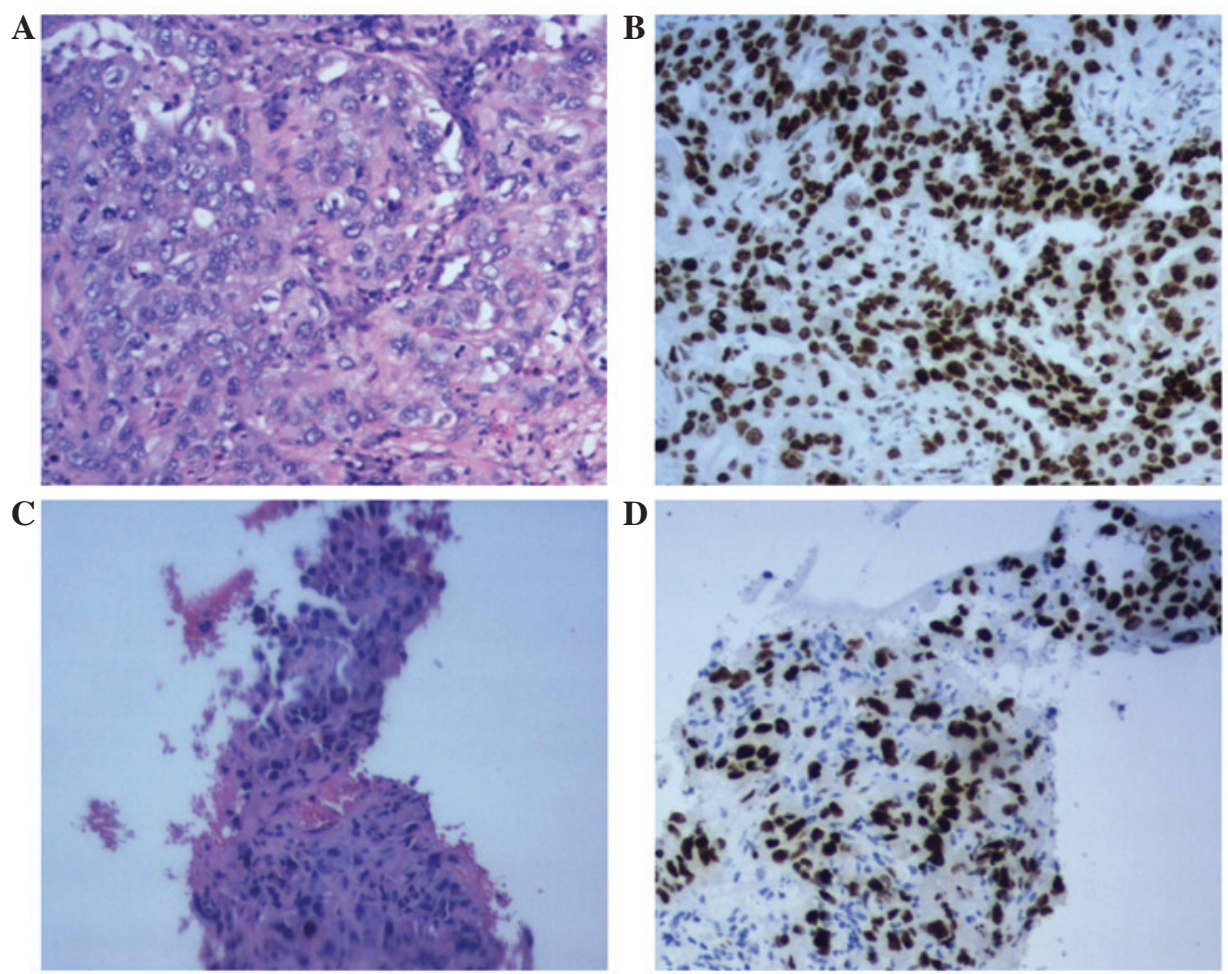

Figure 1. Case one. Hematoxylin and eosin staining of TTF-1 in primary lung tumor and metastatic pancreatic lesions. (A) Adenocarcinoma was identified in the primary lung tumor sample, which was (B) positive for TTF-1. (C) Adenocarcinoma was identified in the metastatic pancreatic lesions, which were (D) positive for TTF-1. Magnification, x400. TTF-1, thyroid transcription factor-1.

(bpm). Laboratory tests revealed the following: Red blood cell (RBC) count, $2.58 \times 10^{12} / 1$ (normal range, $3.68-5.13 \times 10^{12} / 1$ ); hemoglobin concentration, $56 \mathrm{~g} / \mathrm{l}$ (normal range, $114-151 \mathrm{~g} / \mathrm{l})$; hematocrit, 19.7\% (normal range, 33.5-45.0\%). White blood cell (WBC) count $\left(5.9 \times 10^{9} / 1\right)$, platelet count $\left(304 \times 10^{9} / 1\right)$, blood urea nitrogen (BUN) levels $(5.7 \mathrm{mmol} / \mathrm{l})$ and creatinine $(\mathrm{Cr})$ levels $(68 \mu \mathrm{mol} / \mathrm{l})$ were all normal. Furthermore, the serum alanine aminotransferase (ALT; $103 \mathrm{U} / 1$; normal range, 5-40 U/1), serum aspartate aminotransferase (AST; $61 \mathrm{U} / 1$; normal range, 8-40 IU/l), serum $\gamma$-glutamyl transpeptidase $(\gamma$-GT; $508 \mathrm{U} / 1$; normal range, 11-50 IU/1) and serum alkaline phosphatase (AKP; $320 \mathrm{U} / 1$; normal range, 40-150 U/l) levels were elevated. In addition, the general bilirubin $(40 \mu \mathrm{mol} / \mathrm{l}$; normal range, 9-21 $\mu \mathrm{mol} / \mathrm{l})$, direct bilirubin $(22 \mu \mathrm{mol} / \mathrm{l}$; normal range, $0-5 \mu \mathrm{mol} / \mathrm{l})$ and total bile acid $(41 \mu \mathrm{mol}$; normal range, $1-12 \mu \mathrm{mol} / \mathrm{l})$ levels were elevated. Tumor markers, including $\alpha$-fetoprotein $(\alpha-F P)$, carcinoembryonic antigen (CEA), carcinoma antigen (CA)19-9, CA-125 and prostate-specific antigen (PSA) were all within normal limits. Coagulation tests were also normal. In addition, the fecal occult blood test was strongly positive.

GI bleeding and obstructive jaundice was suspected. A small bowel endoscopy revealed a large amount of blood in the upper jejunum, however, no active bleeding sites were identified. Abdominal contrast-enhanced computed tomography (CT) revealed multiple lesions in the pancreas (Fig. 2A and $\mathrm{B}$ ), and distention of the intra- and extrabiliary ducts. Enlarged peripancreatic lymph nodes were also detected. A subsequent endoscopic retrograde cholangiopancreatography revealed stenosis of the distal common bile duct and thus, a plastic stent was inserted into the common bile duct. A week after the insertion procedure, the AST, ALT, AKP, $\gamma$-GT, bilirubin and total bile acid levels were normal. However, the patient's WBC count was elevated to $20.5 \times 10^{9} / 1$ and the hemoglobin level was $68 \mathrm{~g} / \mathrm{l}$, even after multiple RBC blood transfusions.

In October 2013, ultrasonography-guided biopsy of one lesion surrounding the pancreas confirmed a diagnosis of adenocarcinoma (Fig. 1C), and the immunohistochemistry results were consistent with primary lung cancer [TTF-1 ${ }^{+}$ (Fig. 1D), $\mathrm{CK}^{+}$, p63- ${ }^{-} \mathrm{CK}^{-}$and CK6-]. The epidermal growth factor receptor (EGFR) mutation test revealed no mutation of the tumor. The diagnosis of lung adenocarcinoma with metastasis to the pancreas was confirmed. As the tumor was not resectable, surgeons recommended conservative treatment. The patient survived for six months following the resection of the primary lung cancer and six weeks after the diagnosis of metastatic pancreatic cancer from NSCLC. However, in December 2013, the patient succumbed to respiratory failure.

Case two. In July 2013, a 63-year-old male presented to The First Affiliated Hospital of Zhejiang University, with melena and weight loss of $2 \mathrm{~kg}$ over a two-month period. The patient had no history of smoking. In May 2010, the patient was diagnosed with early-stage lung adenocarcinoma and subsequently underwent right upper lung lobectomy via a thoracotomy. The patient received no adjuvant chemotherapy or radiotherapy. In July 2011, a craniotomy was performed for the resection of a solitary brain metastasis from NSCLC. No detailed medical data is available for the two surgical procedures, as they were performed at another hospital.

The patient was referred to the Hepatobiliary and Pancreatic Unit of The First Affiliated Hospital of Zhejiang University (July, 
A

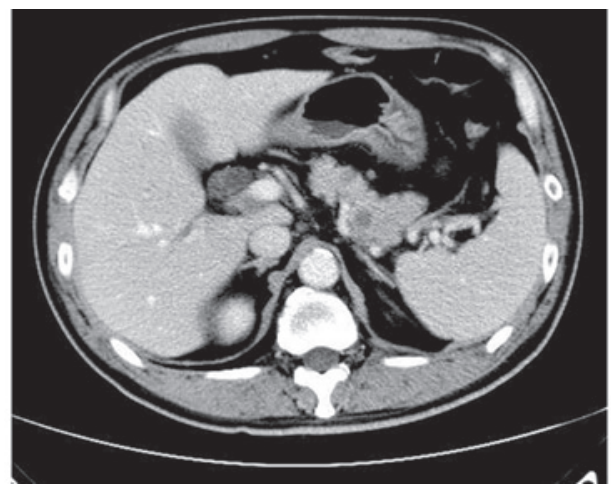

B

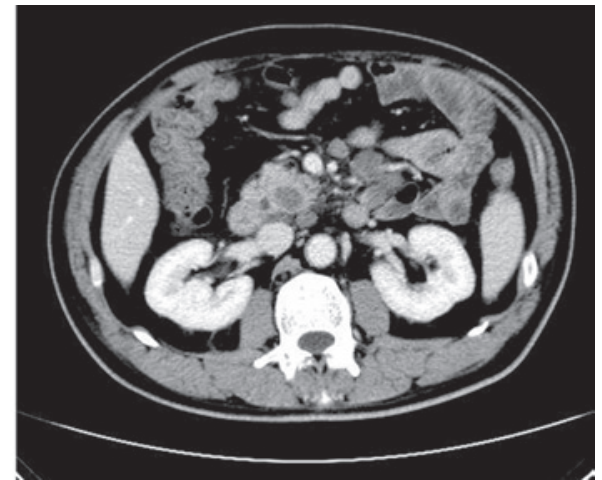

Figure 2. Case one. Abdominal contrast-enhanced computed tomography revealing a hypodense mass in the (A) tail and (B) head of the pancreas.
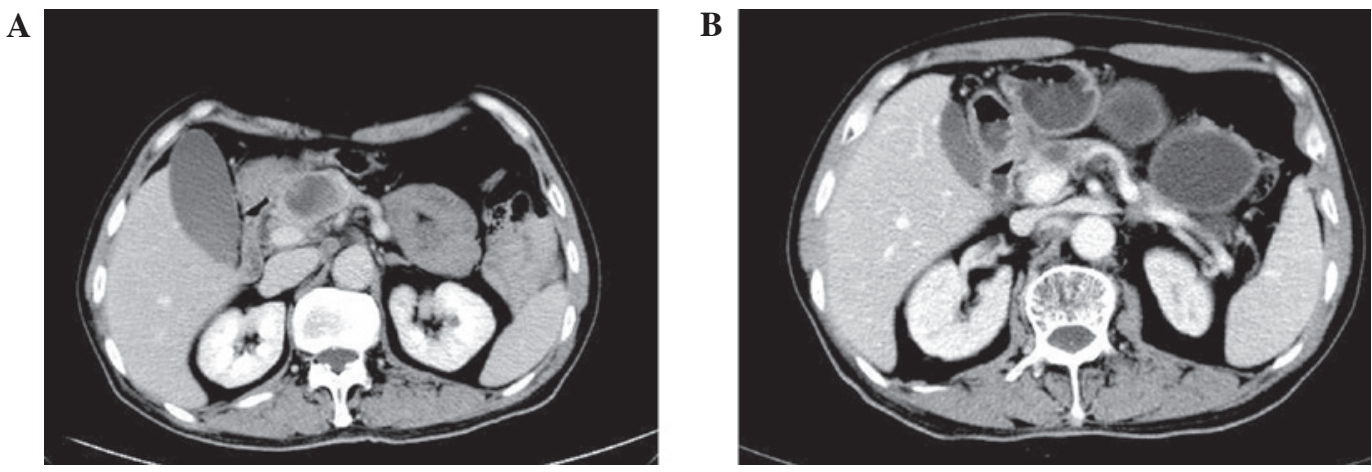

Figure 3. Case two. Abdominal enhanced computed tomography scan showing (A) a mass located in the head of pancreas, which exhibited (B) evident shrinkage following two cycles of chemotherapy.
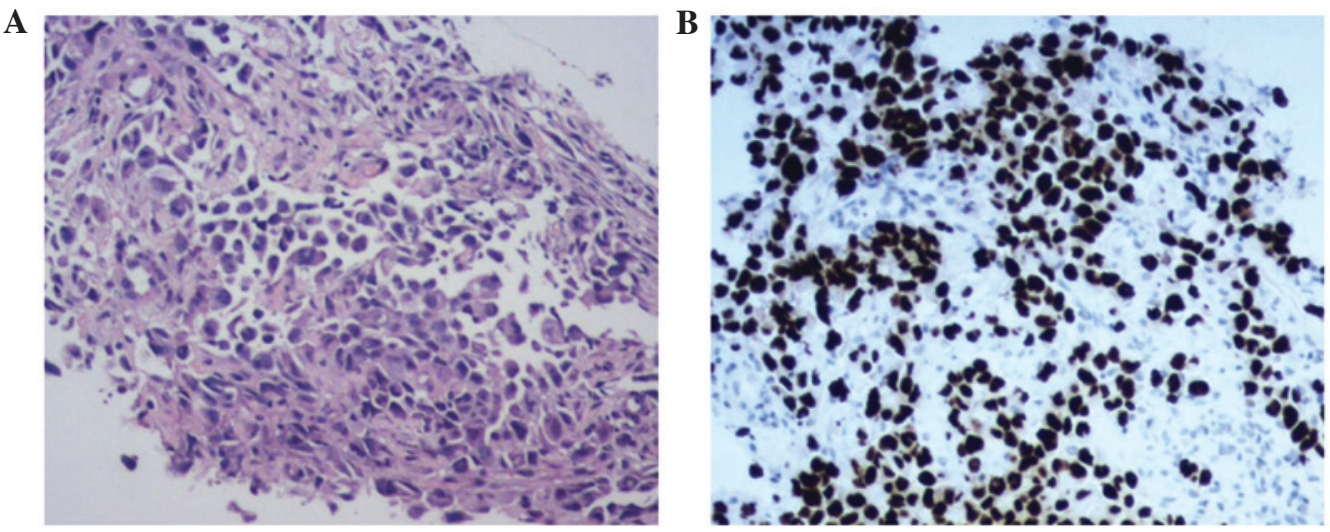

Figure 4. Case two. Staining for (A) hematoxylin and eosin (magnification, $\mathrm{x} 400$ ) and (B) thyroid transcription factor-1 in the pancreatic lesions (magnification, $\mathrm{x} 400$ ).

2013). Upon physical examination, the patient was pale with no apparent pulmonary distress or dizziness. Epigastric tenderness was noted and a stool sample was found to be strongly positive in a fecal occult blood test. The patient's blood pressure was $99 / 54 \mathrm{mmHg}$, with a pulse rate of $87 \mathrm{bpm}$. Laboratory examinations revealed a blood hemoglobin level of $66 \mathrm{~g} / \mathrm{l}$ and a hematocrit level of $21.6 \%$. Other tests, including ALT, AST, AKP, $\gamma$-GT, bilirubin, total bile acid, BUN, Cr, WBC count, platelet, CEA, $\alpha-\mathrm{FP}, \mathrm{CA} 19-9, \mathrm{CA}-125$, PSA and coagulation tests, were unremarkable. Upper GI bleeding was suspected.

An enhanced abdominal CT scan revealed a mass that was located in the head of the pancreas (Fig. 3A), with enlargement of the retroperitoneal lymph nodes, which indicated malignancy. Biliary tract dilation was not detected. In August 2013, a fine-needle ultrasonography-guided biopsy of the pancreatic head lesion revealed adenocarcinoma (Fig. 4A). Immunostaining confirmed that the tumor originated from the lung, as staining was positive for CK, TTF-1 (Fig. 4B) and NapsinA, but negative for CK7, CDX2 and CK5/6. A EGFR mutation test confirmed that the tumor was wild-type EGFR. Subsequently, the patient was administered systemic chemotherapy with paclitaxel $\left(175 \mathrm{mg} / \mathrm{m}^{2}\right.$, day 1) and cisplatin (25 mg/m², days 1-3) every three weeks for four cycles. After two cycles of chemotherapy, an additional 
abdominal CT scan revealed shrinkage of the pancreatic lesion (Fig. 3B). Melena was no longer present and a repeated fecal occult blood test was negative. Furthermore, the blood hemoglobin level had increased to $79 \mathrm{~g} / \mathrm{l}$. The patient continued chemotherapy $\left(175 \mathrm{mg} / \mathrm{m}^{2}\right.$ paclitaxel, day 1 ; and $25 \mathrm{mg} / \mathrm{m}^{2}$ cisplatin, days $1-3$, every 3 weeks) for an additional two cycles. However, treatment was interrupted when the patient suffered a stroke. Subsequently, chemotherapy was stopped. The patient succumbed to NSCLC in December, 2014.

\section{Discussion}

Overall, $>50 \%$ of cases of pancreatic metastases are asymptomatic and thus, are detected during follow-up investigations after the diagnosis of the primary lesion (9). Symptomatic metastatic lesions of the pancreas from lung cancer are rare (10). Pancreatic metastatic lesions may directly invade the pancreatic duct epithelium, mimicking primary pancreatic cancer clinically. Similar to primary pancreatic carcinoma, the majority of symptoms caused by metastatic pancreatic lesions are not organ-specific, including weight loss, fatigue, abdominal or back pain, nausea and jaundice (11). In the current study, the two patients presented with weight loss. The most common symptoms of pancreatic metastasis from lung cancer that have been reported in the literature are obstructive jaundice and acute pancreatitis $(12,13)$. Z'Graggen et al (6) reported the first case of GI bleeding following solitary metastasis from lung carcinoma in the head of the pancreas in 1998 (6). At present, only three cases, including the present two cases and that reported by Z'Graggen et al (6), of GI bleeding due to pancreatic metastasis from lung cancer have been reported in the English literature. Metastatic lesions of the pancreas from lung cancer that cause GI bleeding are much less common. GI bleeding may cause anemia, as well as occult blood in the stool, which was identified in the two cases presented in the current study.

The clinical diagnosis of metastatic pancreatic tumors is difficult. Imaging studies may aid with the differential diagnosis between lung cancer and pancreatic cancer in patients with a pancreatic mass and a history of malignant lung neoplasm (14-16). A history of lung cancer may lead to the diagnosis of pancreatic metastasis. As was observed in the two cases of the present study, peripancreatic lymph node involvement and multiple tumors may result from metastatic lung carcinoma (17). By contrast to primary pancreatic cancers, which are usually relatively hypo-vascular, highly vascular tumors identified in imaging studies are more likely to be metastases (6), which was not indicated in the present two patients. However, patient medical history and imaging alone cannot lead to a definitive diagnosis, which requires pathological examination of tissue samples. Immunohistochemical techniques provide an improved method of distinction. TTF-1 is positive in $~ 76 \%$ of lung adenocarcinomas and CK7 is frequently reactive in both lung and pancreatic cancers (18). In the present study, the two patients were each positive for TTF-1, but case one was positive for CK7 and case two was negative. The majority of pancreatic cancers stain positively for CK20, which may provide valuable delineation (14). Therefore, immunostaining of TTF-1 and/or CK20 must be performed in these patients.
NSCLC with pancreatic metastasis is classified as stage IV disease according to the AJCC TNM staging system (7) and has a poor prognosis. However, when patients with solitary metastasis to the pancreas are carefully evaluated and selected, they become candidates for pancreatic resection, with certain patients achieving long-term survival $(6,12,19)$. As in the present cases, the majority of lung cancer patients suffer from widespread disease that is unresectable (20). Platinum-based chemotherapy has been shown to improve survival and quality of life. Chemotherapy is most suitable for patients with a good performance status. Liu et al (13) studied 20 patients with lung cancer metastasis-induced acute pancreatitis, of which 15 cases were from NSCLC and five were from small cell lung cancer (13). It was found that the group treated with chemotherapy exhibited a longer mean survival time (161.9 days) than those without chemotherapy treatment $(25.0$ days $)(\mathrm{P}<0.01)$. The study hypothesized that chemotherapy may prolong survival time of select patients with metastasis-induced acute pancreatitis from lung cancer (13). In the present study, the second patient received chemotherapy with paclitaxel and carboplatin; the GI bleeding subsequently stopped and the patient's blood hemoglobin level increased. By contrast, the first patient did not receive chemotherapy and succumbed soon after. We hypothesize that appropriate patients with advanced NSCLC involving the pancreas must receive chemotherapy or other effective treatments. Recently, newly developed target agents have shown efficacy in certain subtypes of lung cancer. For example, patients with sensitive EGFR gene mutations may benefit from treatment with EGFR tyrosine-kinase inhibitors, including gefitinib and erlotinib (21). Furthermore, the anaplastic lymphoma kinase (ALK) tyrosine-kinase inhibitor, crizotinib, exhibited marked antitumor activity in patients with advanced NSCLC with ALK gene rearrangements (22). However, in the present study, both patients were wild-type EGFR and the ALK gene test was not performed. Thus, the patients did not receive treatment with EGFR or ALK tyrosine-kinase inhibitors.

In conclusion, upper GI bleeding caused by pancreatic metastasis of lung cancer is extremely rare. Chemotherapy may stop GI bleeding and prolong patient survival, and clinicians must consequently be aware of this manifestation. When patients are resistant to conservative therapies, surgery is recommended.

\section{Acknowledgements}

This study was supported by grants from the Zhejiang Provincial Natural Science Fund (grant no. LY13H160007) and the Zhejiang Medicines and Health Science and Technology Project (grant no. 201348801). The authors would like to thank the patients involved and their family members.

\section{References}

1. Breathnach OS, Freidlin B, Conley B, et al: Twenty-two years of phase III trials for patients with advanced non-small-cell lung cancer: sobering results. J Clin Oncol 19: 1734-1742, 2001.

2. Quint LE, Tummala S, Brisson LJ, et al: Distribution of distant metastases from newly diagnosed non-small cell lung cancer. Ann Thorac Surg 62: 246-250, 1996.

3. Line DH and Deeley TJ: The necropsy findings in carcinoma of the bronchus. Br J Dis Chest 65: 238-242, 1971. 
4. Nakamura E, Shimizu M, Itoh T and Manabe T: Secondary tumors of the pancreas: clinicopathological study of 103 autopsy cases of Japanese patients. Pathol Int 51: 686-690, 2001.

5. Adsay NV, Andea A, Basturk O, Kilinc N, Nassar H and Cheng JD: Secondary tumors of the pancreas: an analysis of a surgical and autopsy database and review of the literature. Virchows Arch 444 527-535, 2004.

6. Z'Graggen K, Fernández-del Castillo C, Rattner DW, Sigala H and Warshaw AL: Metastases to the pancreas and their surgical extirpation. Arch Surg 133: 413-417; discussion 418-419, 1998

7. Edge SB, Byrd DR, Compton CC, Fritz AG, Greene FL and Trotti A (eds): American Joint Committee on Cancer (AJCC) Cancer Staging Manual. 7th edition. Springer, Inc., Chicago, IL, 2010.

8. Oken MM, Creech RH, Tormey DC, et al: Toxicity and response criteria of the Eastern Cooperative Oncology Group. Am J Clin Oncol 5: 649-655, 1982.

9. Zerbi A and Pecorelli N: Pancreatic metastases: An increasing clinical entity. World J Gastrointest Surg 2: 255-259, 2010

10. Bestari MB and Agustanti N: Obstructive jaundice due to pancreatic metastasis from non-small cell lung cancer. Acta Med Indones 45: 216-219, 2013.

11. Merkle EM, Boaz T, Kolokythas O, Haaga JR, Lewin JS and Brambs HJ: Metastases to the pancreas. Br J Radiol 71: $1208-1214,1998$.

12. Pericleous S, Mukherjee S and Hutchins RR: Lung adenocarcinoma presenting as obstructive jaundice: a case report and review of literature. World J Surg Oncol 6: 120, 2008.

13. Liu SF, Zhang S, Chen YC, et al: Experience of cancer care for metastasis-induced acute pancreatitis patients with lung cancer. J Thorac Oncol 4: 1231-1235, 2009.
14. Schwarz RE, Chu PG and Grannis FW Jr: Pancreatic tumors in patients with lung malignancies: a spectrum of clinicopathologic considerations. South Med J 97: 811-815, 2004.

15. Sato M, Okumura T, Kaito K, et al: Usefulness of FDG-PET/ $\mathrm{CT}$ in the detection of pancreatic metastases from lung cancer. Ann Nucl Med 23: 49-57, 2009.

16. Xi-wen S and Shu-zhen C: Characterization of pancreatic metastases from primary lung cancer using magnetic resonance imaging. Clin Imaging 34: 351-354, 2010.

17. Onuigbo WI: Lung cancer metastasis to the pancreas and its surrounding lymph nodes. Br J Dis Chest 60: 152-155, 1966.

18. Harlamert HA, Mira J, Bejarano PA, et al: Thyroid transcription factor- 1 and cytokeratins 7 and 20 in pulmonary and breast carcinoma. Acta Cytol 42: 1382-1388, 1998.

19. Mori N, Sawada T, Satoh H, Kawaguchi M, Hara H and Matsushita K: A resected case of solitary pancreatic metastasis from adenocarcinoma of the lung. JOP 9: 698-703, 2008.

20. Maeno T, Satoh H, Ishikawa H, et al: Patterns of pancreatic metastasis from lung cancer. Anticancer Res 18: 2881-2884, 1998.

21. Mok T, Yang JJ and Lam KC: Treating patients with EGFR-sensitizing mutations: first line or second line - is there a difference? J Clin Oncol 31: 1081-1088, 2013

22. Shaw AT, Yeap BY, Solomon BJ, et al: Effect of crizotinib on overall survival in patients with advanced non-small-cell lung cancer harbouring ALK gene rearrangement: a retrospective analysis. Lancet Oncol 12: 1004-1012, 2011. 